\title{
Serous and mucinous ovarian tumors express different profiles of MMP-2, -7, -9, MT1-MMP, and TIMP-1 and -2
}

\author{
JEAN-LUC BRUN $^{1}$, ANNIE CORTEZ ${ }^{2}$, FRÉDÉRIC COMMO ${ }^{2}$, SERGE UZAN ${ }^{1,3}$ \\ ROMAN ROUZIER ${ }^{1,3^{*}}$ and EMILE DARAII ${ }^{1,3 *}$ \\ Departments of ${ }^{1}$ Gynecology and ${ }^{2}$ Pathology, AP-HP, Hôpital Tenon, 4 rue de la Chine, \\ F-75571 Paris Cedex; ${ }^{3}$ UPMC University Paris 06, UPRES EA 4053, F-75005 Paris, France
}

Received June 30, 2008; Accepted September 2, 2008

DOI: 10.3892/ijo_00000114

\begin{abstract}
Matrix metalloproteinases (MMPs) and their tissue inhibitors (TIMPs) play key roles in tumorigenesis, but little is known of their expression according to mucinous or serous type. This study aimed to evaluate the immunohistochemical expression of MMP-2, -7, -9, MT1-MMP, TIMP-1 and -2 in these tumors. A tissue microarray was set up including 99 serous ( 25 benign, 27 borderline, 47 malignant) and 79 mucinous ( 25 benign, 44 borderline, 10 malignant) ovarian tumors. Immunostaining results were scored by using the HSCORE and assessed by univariate, unsupervised hierarchical clustering and multidimensional scaling analyses. Epithelial expression of MMP-2, -7, -9, MT1-MMP, TIMP-2, but not TIMP-1, was higher in serous than mucinous tumors. Stromal expression of MMP-7 was higher in serous tumors. Alterations in MT1-MMP, MMP-7 and -9 were found in malignant serous tumors, while benign and borderline tumors shared similar expressions. By unsupervised hierarchical clustering analysis, mucinous and serous tumors were better differentiated by epithelial than stromal MMP and TIMP immunolabelling. By multidimensional scaling analysis, the expressions of MMPs and TIMPs were scattered in serous tumors and homogeneous for mucinous tumors. In conclusion, our results support the differential expression in MMPs and TIMPs of ovarian tumors according to serous or mucinous histology.
\end{abstract}

\section{Introduction}

Matrix metalloproteinases (MMPs) and their natural inhibitors (TIMPs) act in concert to control extracellular matrix turnover. MMPs and TIMPs have multiple functions during embryogenesis, organogenesis, angiogenesis and wound healing (1-4). MMP and TIMP expression is altered in both

Correspondence to: Dr Jean-Luc Brun, Service de GynécologieObstétrique, Hôpital Tenon, 4 rue de la Chine, 75020 Paris, France E-mail: jean-luc.brun@tnn.aphp.fr

*Co-senior author

Key words: MMP, immunohistochemistry, tissue microarray, serous, mucinous, ovarian neoplasms benign and malignant tumors, and also in invasion and metastasis requiring the breakdown and removal of extracellular matrix $(5,6)$.

MMP-2 and its inhibitor TIMP-2 have been found to control type IV collagen that is the major component of the basement membrane $(7,8)$. MMP-2 is secreted as a proenzyme (proMMP-2) that is mainly produced by stromal fibroblasts (9). MT1-MMP, also called MMP-14, has been found to activate proMMP-2 and to be overexpressed in malignant tumors $(10,11)$. Moreover, MT1-MMP forms a complex with TIMP-2 and serves as a receptor for MMP-2 forming a tri-molecular complex (8). MMP-9 has also the ability to degrade type IV collagen and plays a proteolytic role in tumor invasion that is inhibited by TIMP-1 (12). An imbalance in the production of MMP-9 and TIMP-1 is thought to control extracellular-matrix turnover and inhibition of tumor invasion and metastasis $(13,14)$. MMP-7 also activates proMMP-2 and proMMP-9 and thereby facilitates tumor invasion and metastasis $(15,16)$.

MMP-2, -7, -9, MT1-MMP, TIMP-1 and -2 have often been evaluated separately in ovarian cancers, but little is known of their concomitant expression in benign, borderline and malignant ovarian tumors. Borderline tumors in particular have only been studied in small series $(17,18)$. Moreover, there is a lack of data on the expression of these MMPs and TIMPs according to histological types (mucinous versus serous) (19). We were therefore prompted to analyze the expression of MMP-2, -7, -9, MT1-MMP, TIMP-1 and -2 according to the mucinous and serous histological type in a large series of benign, borderline and malignant ovarian tumors.

\section{Materials and methods}

Patients. Tissue samples were obtained from 178 patients undergoing surgery in the Gynaecology Department of Tenon Hospital, Paris, from 2001 to 2006. The samples consisted of 50 benign ovarian tumors ( 25 serous, 25 mucinous), 71 borderline ovarian tumors (27 serous, 44 mucinous), and 57 malignant ovarian tumors (47 serous, 10 mucinous).

In the benign tumor group, the patients' median age was 48 years (range 20-79): 28 women (56\%) were premenopausal. In the borderline tumor group, the median age 
of patients was 47 years (range 18-84) and 37 women (52\%) were pre-menopausal. The disease was staged according to the International Federation of Gynecology and Obstetrics (FIGO) classification (20). Sixty-four patients had stage I disease, 2 had stage II disease, and 5 had stage III disease. In the malignant tumor group, the median age of patients was 60 years (range 22-90): 14 women (25\%) were pre-menopausal. The FIGO stages were I, II, III or IV in 9 patients, 3 patients, 41 patients and 4 patients, respectively.

All the tumors were reviewed to confirm histological diagnosis. Histological typing followed the FIGO recommendations (20). Borderline ovarian tumors were defined by serous or mucinous tumors, with proliferating activity of the epithelial cells and nuclear abnormalities, but with no infiltrative destructive growth.

Tissue microarray (TMA). Formalin-fixed, paraffinembedded tumor samples were used to construct a TMA (21). Hematoxylin-eosin stained sections were carefully examined to select representative tumor regions from each selected primary tumor block (donor blocks).

Tissue cylinders $(0.6 \mathrm{~mm}$ in diameter $)$ were punched from the region of the donor block with the use of a custom-made precision instrument (Beecher Instruments, Silver Spring, MD) and transferred to a $25 \mathrm{~mm}$ x $35 \mathrm{~mm}$ paraffin block under microscopic control. Each specimen was sampled three times to avoid tissue loss and to optimize representation of tumors (22). Four tissue cores sampled from endometrial cancers were arranged at one corner of the block for the purpose of positive control and proper orientation of the TMA block.

TMA blocks were incubated at $56^{\circ} \mathrm{C}$ for $5 \mathrm{~min}$ to allow recipient and donor paraffin to stick together before cutting and cooled slowly to room temperature. They were cut into $4 \mu \mathrm{m}$ sections and transferred to glass slides. Separate sections from the TMA blocks were used for immunohistochemical analysis.

Immunohistochemistry. Tissues were immediately fixed in formalin (10\%) and then processed as paraffin blocks. Four-micron-thick sections of formalin-fixed tissues were deparaffinated in xylene and rehydrated through a graded series of ethanol solutions. Sections were immunostained using the Ventana Nexes automated immunohistochemistry system (Ventana Medical Systems, Tucson, AZ).

Purified mouse monoclonal or rabbit polyclonal antibodies against human MMP-2,-7,-9, MT1-MMP, TIMP-1, and -2 were used as primary antibodies at various concentrations: MMP-2 (mouse, clone 42-5D11; Calbiochem, San Diego, CA; $5 \mu \mathrm{g} / \mathrm{ml}$ ), MMP-7 (mouse, clone ID2; Lab Vision Corp., Fremont, CA; $1.3 \mu \mathrm{g} / \mathrm{ml}$ ), MMP-9 (mouse, clone 562A4; Calbiochem; $20 \mu \mathrm{g} / \mathrm{ml}$ ), MT1-MMP (rabbit; Lab Vision Corp.; $8 \mu \mathrm{g} / \mathrm{ml}$ ), TIMP-1 (mouse, clone 102D1; Lab Vision Corp.; $8 \mu \mathrm{g} / \mathrm{ml}$ ), and TIMP-2 (mouse, clone 3A4; Lab Vision Corp.; $4 \mu \mathrm{g} / \mathrm{ml}$ ).

Prior to the primary antibody staining, an antigen retrieval step was used, combined with a high temperature antigenunmasking technique (Dako Target Retrieval Solution, Glostrup, Denmark; $100^{\circ} \mathrm{C}, 30 \mathrm{~min}$ ). For MMP-7, antigen unmasking was achieved with proteinase $\mathrm{K}, 4 \mathrm{~min}$. The auto- mated procedure is based on an indirect biotin-avidin system with a universal biotinylated immunoglobulin as secondary antibody, diaminobenzidine as substrate, and hematoxylin as counterstain. Except for MT1-MMP, a Ventana amplification kit was used in addition to the automated procedure (Ventana Medical Systems).

Positive controls for MMP-2, MMP-7, MMP-9, MT1MMP, TIMP-1 and TIMP-2 were sections of endometrial cancers which had been strongly stained in a previous study (16). For negative control, the primary antibody was replaced by an irrelevant non-immune mouse antibody of the same immunoglobulin G subtype.

Semi-quantitative analysis. The TMA was analyzed by light microscopy by use of a $10 x$ objective. Immunostaining results were scored by JLB and AC independently, using the HSCORE (23). The HSCORE was produced by multiplying the percentage of stained tumor cells (0-100\%) with the intensity score. The intensity of staining was scored on a 4point scale: 0 , no staining; 1 , weak; 2 , moderate; 3 , intense. Thus, each score ranged from 0 to 300 . For each tumor specimen, the HSCORE of a given MMP or TIMP was assessed in epithelial and stromal cells. Discordance between the two examiners never exceeded $5 \%$.

Statistical analysis. All analyses were performed using the $\mathrm{R}$ package with the libraries and gplots (http://lib.stat.cmu. edu/R/CRAN/). Parametric and non-parametric continuous variables were compared with Student's t-test. ANOVA was used when multiple groups were compared, and selected pair comparisons were performed using Tukey's 'honest significant difference' method. Correlations were evaluated with the kappa coefficient. P-values $\leq 0.05$ were considered statistically significant. Unsupervised hierarchical clustering analysis was used to organize the results of immunostaining into meaningful structures, applying the same approach that has previously been adopted for cDNA micro-arrays and has also been applied to TMA data (24-26). Clustering analysis organizes cases according to the similarity or dissimilarity of immunostaining profiles, placing the cases with similar immunoprofiles together as neighboring rows in the clustergram. The relationship between cases and immunomarkers is depicted graphically as a dendrogram in which branch length is determined by the correlation between immunostaining results. All HSCORE data for each biomarker were used for clustering analysis. Only cases with immunostaining data for all markers under consideration were entered into clustering analysis. Complete linkage hierarchical clustering was done with Euclidean distance as distance metric. Data were centered and scaled in the row direction (samples). Tumors clustering together in main clusters and histological type were compared using the Mac Nemar test.

Classical Multidimensional Scaling (MDS) was performed to visually assess distances between tumors. This procedure, also known as principal component analysis, takes a matrix of interpoint distances and creates a configuration of points (one point representing one tumor). These points are constructed in two dimensions, and the Euclidean distances between them approximately reproduce the original distance matrix. Thus, a 
Table I. Expression (mean \pm SD) of MMPs and TIMPs in serous and mucinous ovarian tumors.

\begin{tabular}{lcccccc}
\hline & \multicolumn{2}{c}{ Epithelial signal } & & \multicolumn{2}{c}{ Stromal signal } \\
\cline { 2 - 3 } & $\begin{array}{c}\text { Serous } \\
(\mathrm{n}=99)\end{array}$ & $\begin{array}{c}\text { Mucinous } \\
(\mathrm{n}=79)\end{array}$ & P-value & $\begin{array}{c}\text { Serous } \\
(\mathrm{n}=99)\end{array}$ & $\begin{array}{c}\text { Mucinous } \\
(\mathrm{n}=79)\end{array}$ & $\begin{array}{c}\text { P-value } \\
\end{array}$ \\
\hline MMP-2 & $94.9 \pm 49.5$ & $52.5 \pm 42.2$ & $<0.001$ & $34.6 \pm 37.7$ & $26.5 \pm 27.4$ & $\mathrm{NS}$ \\
MMP-7 & $124.1 \pm 88.1$ & $62.0 \pm 54.4$ & $<0.001$ & $14.5 \pm 18.6$ & $32.8 \pm 30.7$ & $<0.001$ \\
MMP-9 & $111.6 \pm 50.1$ & $58.3 \pm 53.2$ & $<0.001$ & $18.6 \pm 21.4$ & $18.3 \pm 23.5$ & $\mathrm{NS}$ \\
MT1-MMP & $115.3 \pm 66.2$ & $43.7 \pm 31.0$ & $<0.001$ & $10.7 \pm 21.8$ & $8.8 \pm 10.2$ & $\mathrm{NS}$ \\
TIMP-1 & $85.7 \pm 41.8$ & $105.9 \pm 61.1$ & $\mathrm{NS}$ & $10.3 \pm 12.3$ & $11.6 \pm 11.5$ & $\mathrm{NS}$ \\
TIMP-2 & $143.1 \pm 54.8$ & $98.0 \pm 58.2$ & $<0.001$ & $27.4 \pm 23.9$ & $26.9 \pm 22.6$ & $\mathrm{NS}$ \\
\hline
\end{tabular}

NS, not significant.

Table II. Expression (mean \pm SD) of MMPs and TIMPs in serous ovarian tumors.

\begin{tabular}{|c|c|c|c|c|c|c|c|c|}
\hline & \multicolumn{3}{|c|}{ Serous epithelial signal } & \multirow[b]{2}{*}{ P-value } & \multicolumn{3}{|c|}{ Serous stromal signal } & \multirow[b]{2}{*}{ P-value } \\
\hline & $\begin{array}{l}\text { Benign } \\
(n=25)\end{array}$ & $\begin{array}{l}\text { Borderline } \\
\qquad(\mathrm{n}=27)\end{array}$ & $\begin{array}{l}\text { Malignant } \\
(\mathrm{n}=47)\end{array}$ & & $\begin{array}{l}\text { Benign } \\
(n=25)\end{array}$ & $\begin{array}{l}\text { Borderline } \\
\quad(n=27)\end{array}$ & $\begin{array}{l}\text { Malignant } \\
\quad(n=47)\end{array}$ & \\
\hline MMP-2 & $123.0 \pm 58.8^{\mathrm{d}}$ & $67.1 \pm 30.4^{\mathrm{a}, \mathrm{d}}$ & $95.8 \pm 45.9^{\mathrm{a}}$ & $<0.001$ & $44.8 \pm 28.5^{\mathrm{a}}$ & $20.2 \pm 21.7^{\mathrm{a}}$ & $37.6 \pm 46.5$ & 0.049 \\
\hline MMP-7 & $166.0 \pm 78.4^{\mathrm{d}}$ & $189.3 \pm 78.2^{\mathrm{c}}$ & $72.5 \pm 62.9^{\mathrm{c}, \mathrm{d}}$ & $<0.001$ & $11.4 \pm 9.9$ & $20.2 \pm 20.9$ & $12.8 \pm 20.3$ & NS \\
\hline MMP-9 & $99.5 \pm 55.2$ & $91.1 \pm 40.3^{\mathrm{a}}$ & $126.1 \pm 48.3^{\mathrm{a}}$ & 0.010 & $14.8 \pm 26.1$ & $12.3 \pm 15.4^{\mathrm{a}}$ & $24.4 \pm 20.6^{\mathrm{a}}$ & 0.040 \\
\hline MT1-MMP & $98.7 \pm 53.1$ & $94.7 \pm 43.1^{\mathrm{a}}$ & $134.1 \pm 76.7^{\mathrm{a}}$ & 0.027 & $15.9 \pm 38.9$ & $3.3 \pm 3.6$ & $12.4 \pm 13.5$ & NS \\
\hline TIMP-1 & $110.1 \pm 44.6^{\mathrm{d}}$ & $90.9 \pm 38.3$ & $71.5 \pm 36.5^{\mathrm{d}}$ & $<0.001$ & $22.7 \pm 14.2^{\mathrm{c}, \mathrm{d}}$ & $7.1 \pm 8.9^{c}$ & $5.7 \pm 7.9^{\mathrm{d}}$ & $<0.001$ \\
\hline TIMP-2 & $156.4 \pm 62.1^{\mathrm{a}}$ & $111.0 \pm 42.7^{\mathrm{a}, \mathrm{b}}$ & $152.1 \pm 51.4^{\mathrm{b}}$ & 0.005 & $41.0 \pm 26.6^{\mathrm{d}}$ & $11.5 \pm 7.6^{\mathrm{b}, \mathrm{d}}$ & $29.6 \pm 23.8^{b}$ & $<0.001$ \\
\hline
\end{tabular}

NS, not significant. ${ }^{\mathrm{a}} \mathrm{p}<0.05 ;{ }^{\mathrm{b}} \mathrm{p}<0.01 ;{ }^{\mathrm{c} \text { or } \mathrm{d}} \mathrm{p}<0.001 ;{ }^{\mathrm{c}, \mathrm{d}} \mathrm{p}<0.001$ twice.

scatter plot of these points provides a visual representation of the original distances between tumors according to MMP expression. As hierarchical clustering, MDS is an unsupervised approach.

\section{Results}

Epithelial expression of MMPs and TIMPs were determined in $92 \%$ (range, 89-94) of tissue samples and stromal expression in 98\% (range, 97-99). Immunostaining data were available for all markers in 83 of the 99 patients (84\%) with serous tumors and in 65 of the 79 patients $(82 \%)$ with mucinous tumors.

MMP and TIMP expression in serous and mucinous ovarian tumors. Serous tumors expressed a higher semi-quantitative epithelial expression of MMP-2, -7, -9, MT1-MMP, and TIMP-2 than mucinous tumors (Table I). TIMP-1 epithelial expression was lower in serous than in mucinous tumors.

Serous tumors expressed a higher semi-quantitative stromal expression of MMP-7 than mucinous tumors. No significant difference in stromal expression of MMP-2, -9,
MT1-MMP, TIMP-1 and TIMP-2 was found between serous and mucinous ovarian tumors.

MMP and TIMP expression in benign, borderline and malignant serous ovarian tumors. The mean expression $( \pm \mathrm{SD})$ of epithelial and stromal cells positive for MMP-2, -7, -9, MT1MMP, TIMP-1 and -2 in serous benign, borderline and malignant tumors are given in Table II.

A difference in semi-quantitative epithelial MMP-2, MMP-7, MMP-9, MT1-MMP, TIMP-1, and TIMP-2 expression was observed among the three tumor groups. Using Student's t-test for MMP-2, the difference was significant between benign and borderline tumors $(\mathrm{p}<0.001)$, and between borderline and malignant tumors $(\mathrm{p}=0.042)$, but not between benign and malignant tumors. MMP-7 epithelial expression was lower in malignant than in benign $(\mathrm{p}<0.001)$ or borderline tumors $(\mathrm{p}<0.001)$ but not different between benign and borderline ovarian tumors.

MMP-9 epithelial expression was higher in malignant than in borderline tumors $(\mathrm{p}=0.016)$ but not different between benign and borderline or malignant ovarian tumors. MT1MMP epithelial expression was higher in malignant than in 


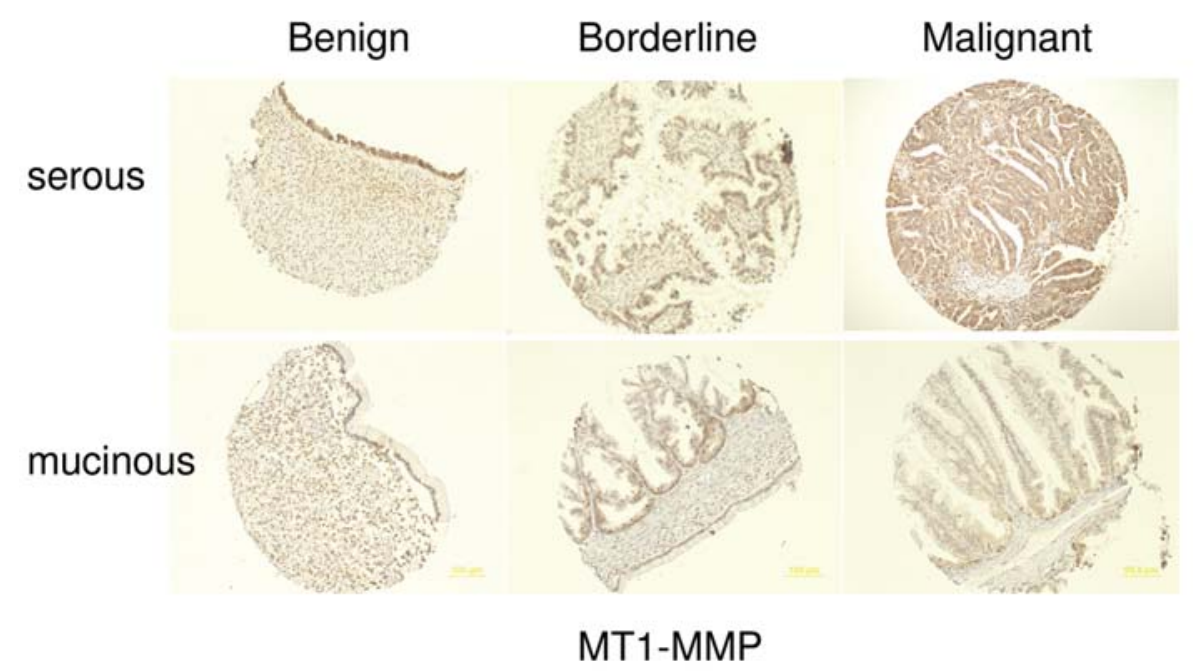

Figure 1. MT1-MMP expression in benign, borderline, malignant serous and mucinous ovarian tumors. Representative cases showing a higher epithelial expression in serous than in mucinous ovarian tumors. In serous tumors, MT1-MMP epithelial expression was higher in malignant than in borderline and benign tumors. In mucinous tumors, MT1-MMP epithelial expression was not significantly different among benign, borderline and malignant tumors; MT1MMP stromal expression was higher in benign than in borderline or malignant tumors.

Table III. Expression (mean \pm SD) of MMPs and TIMPs in mucinous ovarian tumors.

\begin{tabular}{|c|c|c|c|c|c|c|c|c|}
\hline & \multicolumn{3}{|c|}{ Mucinous epithelial signal } & \multirow[b]{2}{*}{ P-value } & \multicolumn{3}{|c|}{ Mucinous stromal signal } & \multirow[b]{2}{*}{ P-value } \\
\hline & $\begin{array}{l}\text { Benign } \\
(n=25)\end{array}$ & $\begin{array}{c}\text { Borderline } \\
\quad(n=44)\end{array}$ & $\begin{array}{l}\text { Malignant } \\
(\mathrm{n}=10)\end{array}$ & & $\begin{array}{l}\text { Benign } \\
(n=25)\end{array}$ & $\begin{array}{l}\text { Borderline } \\
(\mathrm{n}=44)\end{array}$ & $\begin{array}{l}\text { Malignant } \\
(\mathrm{n}=10)\end{array}$ & \\
\hline MMP-2 & $70.7 \pm 22.0^{\mathrm{c}}$ & $31.5 \pm 32.0^{\mathrm{c}, \mathrm{d}}$ & $94.3 \pm 61.0^{\mathrm{d}}$ & $<0.001$ & $46.1 \pm 24.3^{\mathrm{b}, \mathrm{c}}$ & $18.3 \pm 26.2^{\mathrm{c}}$ & $13.6 \pm 9.3^{\mathrm{b}}$ & $<0.001$ \\
\hline MMP-7 & $65.7 \pm 54.3$ & $65.6 \pm 57.4$ & $37.6 \pm 37.4$ & NS & $30.5 \pm 26.2$ & $37.5 \pm 32.8$ & $19.1 \pm 30.0$ & NS \\
\hline MMP-9 & $33.6 \pm 33.2^{\mathrm{c}}$ & $53.4 \pm 45.4^{\mathrm{d}}$ & $130.0 \pm 58.4^{\mathrm{c}, \mathrm{d}}$ & $<0.001$ & $22.4 \pm 23.1$ & $16.5 \pm 25.7$ & $15.9 \pm 13.2$ & NS \\
\hline MT1-MMP & $46.3 \pm 23.9$ & $42.8 \pm 32.9$ & $42.3 \pm 38.1$ & NS & $16.7 \pm 12.7^{\mathrm{a}, \mathrm{c}}$ & $4.7 \pm 5.7^{\mathrm{c}}$ & $7.3 \pm 7.8^{\mathrm{a}}$ & $<0.001$ \\
\hline TIMP-1 & $117.6 \pm 44.1$ & $93.7 \pm 64.7$ & $128.1 \pm 74.7$ & NS & $21.8 \pm 11.9^{\mathrm{c}, \mathrm{d}}$ & $6.6 \pm 7.2^{\mathrm{d}}$ & $7.6 \pm 9.0^{\mathrm{c}}$ & $<0.001$ \\
\hline TIMP-2 & $115.8 \pm 44.3^{\mathrm{a}}$ & $76.1 \pm 53.3^{\mathrm{a}, \mathrm{c}}$ & $149.5 \pm 64.3^{\mathrm{c}}$ & $<0.001$ & $47.8 \pm 24.0^{\mathrm{b}, \mathrm{c}}$ & $14.3 \pm 12.1^{\mathrm{a}, \mathrm{c}}$ & $28.6 \pm 12.6^{\mathrm{a}, \mathrm{b}}$ & $<0.001$ \\
\hline
\end{tabular}

NS, not significant. ${ }^{\mathrm{a}} \mathrm{p}<0.05 ;{ }^{\mathrm{b}} \mathrm{p}<0.01 ;{ }^{\mathrm{c} \text { or }} \mathrm{p}<0.001 ;{ }^{\mathrm{c}, \mathrm{d}} \mathrm{p}<0.001$ twice.

borderline tumors $(\mathrm{p}=0.048)$ but not different between benign and borderline or malignant ovarian tumors (Fig. 1).

TIMP-1 epithelial expression was lower in malignant than in benign tumors $(\mathrm{p}<0.001)$ but not different between borderline and benign or malignant ovarian tumors. TIMP-2 epithelial expression was lower in borderline than in malignant $(p=0.009)$ or benign tumors $(p=0.015)$. No difference in TIMP-2 expression was observed between benign and malignant tumors.

A difference in semi-quantitative stromal MMP-2, MMP-9, TIMP-1, and TIMP-2 expression was observed among the three tumor groups.

Using Student's t-test, MMP-2 stromal expression was higher in benign than in borderline tumors $(\mathrm{p}=0.048)$, while no difference was seen between benign and malignant, or between borderline and malignant tumors.

MMP-9 stromal expression was lower in borderline than in malignant tumors $(\mathrm{p}=0.045)$, while no difference was seen between benign and borderline, or between benign and malig- nant tumors. TIMP-1 stromal expression was higher in benign than in borderline $(\mathrm{p}<0.001)$ or malignant tumors $(\mathrm{p}<0.001)$ but not different between borderline and malignant ovarian tumors. TIMP-2 stromal expression was lower in borderline than in benign $(\mathrm{p}<0.001)$ or malignant tumors $(\mathrm{p}=0.002)$. No difference in TIMP-2 stromal expression was observed between benign and malignant tumors.

MMP and TIMP expression in benign, borderline and malignant mucinous ovarian tumors. The mean expressions $( \pm \mathrm{SD})$ of epithelial and stromal cells positive for MMP-2, -7, -9, MT1-MMP, TIMP-1 and -2 in benign, borderline and malignant mucinous tumors are given in Table III. A difference in semi-quantitative epithelial MMP-2, MMP-9, and TIMP-2 expressions was observed among the three tumor groups.

Using Student's t-test for MMP-2, the difference was significant between benign and borderline tumors $(p<0.001)$, and between borderline and malignant tumors $(\mathrm{p}<0.001)$. 


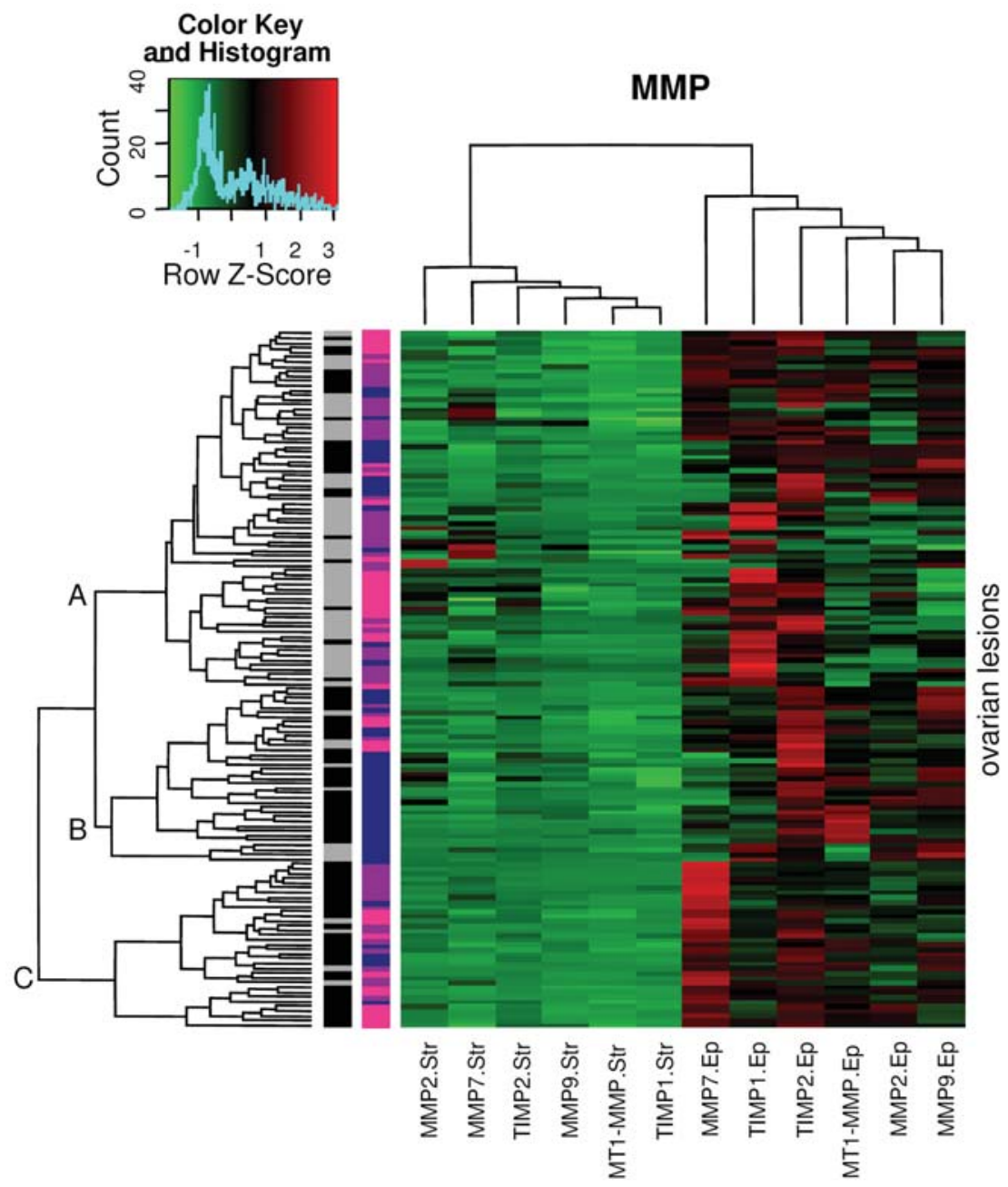

Figure 2. Unsupervised hierarchical clustering analysis of immunostaining data provided by Tissue Micro Array on 148 patients. Rows (samples) and columns (proteins) are arranged according to a hierarchical clustering method. Green and red indicate expression levels respectively below and above the median (black). Rows were also labeled according to histological type (serous in black and mucinous in grey), and according to histology (benign in pink, borderline in purple, and malignant in blue).

No difference in MMP-2 epithelial expression was observed between benign and malignant mucinous tumors. MMP-9 epithelial expression was higher in malignant than in borderline $(\mathrm{p}<0.001)$ or benign tumors $(\mathrm{p}<0.001)$ but not different between benign and borderline ovarian tumors. TIMP-2 epithelial expression was lower in borderline than in benign ( $p=0.012)$ or malignant tumors $(p<0.001)$ but not different between benign and malignant ovarian tumors.

A difference in semi-quantitative stromal MMP-2, MT1MMP, TIMP-1, and TIMP-2 expressions was observed among the three groups of mucinous tumors. MMP-2 stromal expression was higher in benign than in borderline $(\mathrm{p}<0.001)$ or malignant tumors $(\mathrm{p}=0.002)$ but not different between borderline and malignant ovarian tumors.

MT1-MMP stromal expression was higher in benign than in borderline $(\mathrm{p}<0.001)$ or malignant tumors $(\mathrm{p}=0.014)$ but not different between borderline and malignant ovarian tumors (Fig. 1). TIMP-1 stromal expression was higher in benign than in borderline $(\mathrm{p}<0.001)$ or malignant tumors $(\mathrm{p}<0.001)$ but not different between borderline and malignant ovarian tumors. Finally, TIMP-2 stromal expression was higher in benign than in borderline $(\mathrm{p}<0.001)$ or malignant tumors $(\mathrm{p}=0.009)$ and between borderline and malignant tumors $(\mathrm{p}=0.046)$.

Unsupervised hierarchical clustering analysis of immunostaining data. Unsupervised hierarchical clustering analysis was applied to the dataset of MMPs and TIMPs in the 148 patients for whom immunostaining data were available for all markers. This produced a dendrogram with well-defined cluster groups of immunomarkers and cases (Fig. 2). Clear separation of immunomarkers into two distinct groups with large linkage distances was apparent: expression of MMPs and TIMPs were clearly different in stromal and epithelial cells. MMP stromal expressions failed to distinguish ovarian tumors because of their low levels. Three large subsets of hierarchical clustering were distinguished: cluster A included 77 tumors (28 serous and 49 mucinous); cluster B included 36 (24 serous and 12 mucinous); and cluster $\mathrm{C}$ included 35 (31 serous and 4 mucinous). The proportion of serous and mucinous tumors was statistically not similar within these three clusters ( $\mathrm{p}<0.001)$ : $75 \%$ of mucinous tumors (labeled in grey) were in cluster A and $66 \%$ of serous tumors (labeled in black) were in clusters B and C (Fig. 2). 


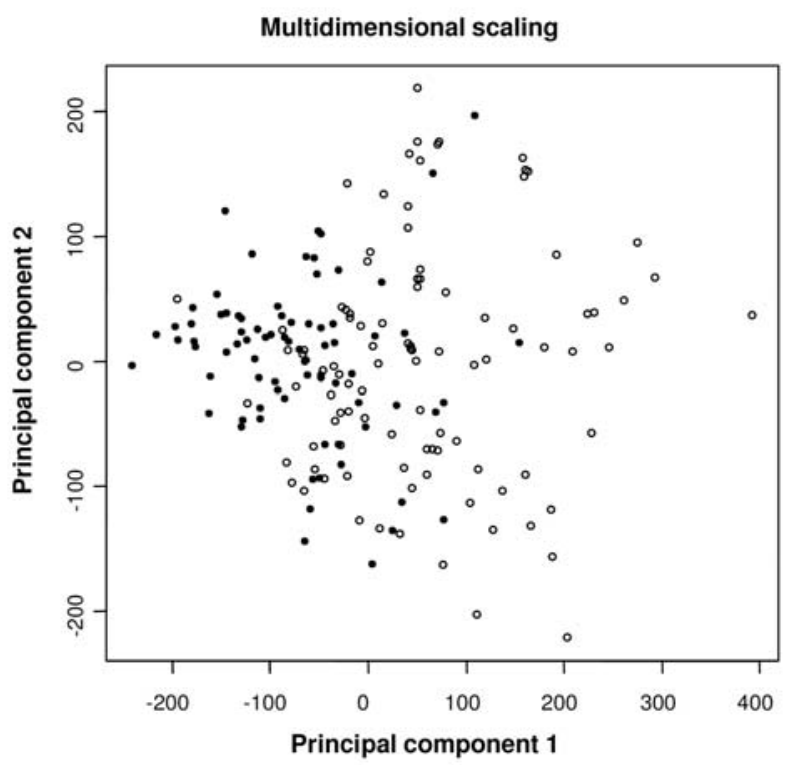

Figure 3. Two dimensional representations of mucinous and serous tumors by multidimensional scaling Mucinous tumors are plotted in black and serous tumors are represented by circles.

Visual analysis of correlation between natural clusters and histology showed that benign and borderline tumors on one side and malignant tumors on the other side tended to cluster together. For example, 30 malignant tumors (labeled in blue) including 23 serous and seven mucinous were found in the 36 samples (83\%) making up cluster B, vs. 15 of the $77(19 \%)$ in cluster A and five of the $35(14 \%)$ in cluster C. Conversely, benign and borderline tumors (labeled in pink and purple respectively) were scattered among each cluster (Fig. 2).

When HSCORES for epithelial MMPs were analyzed as a function of clusters (A, B or C), overexpression of MMP-7 clearly distinguished cluster $\mathrm{C}$, while cluster A was characterized by low expressions of MMP-7, -9, MT1-MMP, and TIMP-2. Expression of TIMP-2 was slightly higher in cluster B compared to cluster $\mathrm{C}$ but not discriminant enough to clearly separate these two clusters.

Multidimensional scaling. Classical MDS showed that mucinous and serous tumors had a different distribution (Fig. 3). Mucinous tumors were grouped together with few outliers indicating that they are a homogeneous entity, while serous tumors had a larger two dimension distribution.

\section{Discussion}

The present study demonstrated that MMPs and TIMPs are differentially expressed in ovarian tumors first according to the mucinous or serous histological type, then according to the benign, borderline or malignant nature of the tumor. Unsupervised analyses by hierarchical clustering analysis and MDS contributed to determining specific MMP and TIMP profiles.

Different expressions of MMP-2, -7, -9, MT1-MMP, and TIMP-1 and -2 have been observed by these two unsu- pervised approaches according to the serous or mucinous histological type of the tumor. Clustering analysis has been widely used to investigate gene expressions by cDNA microarrays in breast cancer, but no such study has been published to date in ovarian tumors $(27,28)$. In our series, serous ovarian tumors had higher epithelial MMP-2, -7, -9, MT1-MMP and TIMP-2 expressions than mucinous tumors, while TIMP-1 expression was higher in mucinous tumors. For stromal MMP and TIMP expression, only MMP-7 was higher in mucinous than in serous ovarian tumors. These data are in keeping with previous studies suggesting that precursors of ovarian epithelial malignancies differ between serous and mucinous tumors (29). In serous neoplasia, ovarian tumors arise from the ovarian surface epithelium or from invagination of the surface epithelium into the ovarian stroma $(30,31)$. In mucinous neoplasia, ovarian tumors are thought to arise from the Müllerian epithelium representing embryological remnants of the Müllerian ducts (29). These data recall those of Fauvet et al demonstrating that apoptosis-related protein expressions differed between serous and mucinous borderline ovarian tumors (32). Moreover, our unsupervised hierarchical clustering analysis clearly showed that mucinous and serous tumors were better differentiated by epithelial than stromal MMPs and TIMPs immunolabelling.

MDS analysis confirmed the scattered distribution of MMPs and TIMPs in serous tumors according to the benign, borderline, or malignant nature, while their expression was homogenous for mucinous tumors. Among the MMPs and TIMPs analyzed in the current study, MT1-MMP, MMP-7 and -9 were the most discriminating factors to assess the nature of ovarian tumors. Alterations in MT1-MMP, MMP-7 and -9 seemed to occur during the transition from borderline to malignant serous tumors, while benign and borderline tumors shared similar expression, suggesting that their alterations correspond to a late step in serous ovarian carcinogenesis. In contrast, Afzal et al showed that borderline and malignant ovarian tumors had similar high MT1-MMP mRNA levels compared to the benign counterpart (33). This apparent discrepancy could be explained by the fact that the study of Afzal et al only included six borderline tumors and that there was no differentiation between serous and mucinous tumors. Our data are in keeping with an in vitro study showing that MT1-MMP expression in ovarian cancer cell lines was linked to matrix degradation and tumor cell invasion, and that MT1MMP could be more inhibited by TIMP-2 than by TIMP-1 $(34,35)$.

In our study, MMP-7 epithelial expression was lower in malignant than in benign or borderline serous tumors, but no difference in MMP-7 expression was observed among mucinous tumors. MMP-7 has been shown to be overexpressed in epithelial ovarian tumors compared to normal ovary, but little is known of MMP-7 expression in borderline and frankly invasive ovarian tumors $(15,19,36)$. Shigemasa et al observed that mucinous tumors expressed similar MMP-7 profiles whatever their benign, borderline or malignant nature (19). In contrast, Wang et al showed that MMP-7 was overexpressed in epithelial ovarian cancer and recombinant MMP-7 promoted invasion in vitro (15). By sense and antisense gene transfection in vitro, they found that MMP-7 overexpression significantly enhanced the invasiveness of 
all MMP-7 stably transfected DOV13 clones. However, in addition to its ability to distinguish the nature of serous ovarian tumors, the low MMP-7 expression in ovarian cancers has been correlated with poor clinical and histological prognostic parameters (36).

As for MMP-7, a higher MMP-9 epithelial expression was observed in malignant than in benign or borderline serous tumors. Our results are in line with those of previous studies reporting a stronger epithelial MMP-9 expression in malignant than in benign or borderline tumors $(37,38)$. Moreover, Zhou et al showed that epidermal growth factor (EGF) cooperated with hepatocyte growth factor (HGF) to promote a highly invasive phenotype via the increased secretion of MMP-9 (39). The coincident induction of MMP-9 was functionally significant because inclusion of MMP-9 inhibitor or an antiMMP-9 neutralizing antibody abolished EGF- and HGFinduced cellular invasion. Recently, Cowden Dahl et al noted that inhibition of polyomavirus enhancer activator 3 (PEA3) expression, a member of a subfamily of Ets domain transcription, reduced the EGF induction of MMP-9 and MT1MMP gene expression, and impaired EGF-stimulated tumor cell invasion (40). These results suggest that elevated PEA3 expression detected in human ovarian cancer may divert cells to a more invasive phenotype by regulating MMP-9 and MMP-14. Symowicz et al suggested a novel mechanism for post-translational modification of E-cadherin function via MMP-9 induction initiated by cell-matrix contact and suggest a mechanism for the promotion of ovarian metastatic dissemination (41). Finally, these results recall those of Darai et al showing that a loss of E-cadherin was observed from benign and borderline to malignant ovarian tumors (42).

In contrast to MT1-MMP, MMP-7 and -9, extensive literature exists on the expression of MMP-2, TIMP-1 and -2 in ovarian tumors. In the current study, MMP-2 differed between benign, borderline and malignant ovarian tumors. However, borderline tumors expressed lower levels of MMP-2 than both benign and malignant tumors. Our data are partly in accordance with those of Sakata et al showing that MMP-2 was more frequently expressed in serous than mucinous malignant tumors (14). Conversely, some studies failed to demonstrate any difference in MMP-2 epithelial expression among epithelial ovarian tumors (17,43-45). These series included a small number of tumors especially of borderline ovarian tumors and MMP profiles were mainly assessed qualitatively $(14,43,44)$. Määttä et al showed than MMP-2 expression was higher in malignant than in borderline or benign ovarian tumors but not different between the two latter groups (17). Differences between our data and those of previous studies can be explained by technical characteristics. Indeed, immunochemistry and mRNA analysis detect both latent and active enzymes, while zymography or Western blot differentiate active MMP-2 from proMMP-2 $(44,45)$. Qi Cai et al found that MMP-2 was expressed more frequently in benign tumors with morphological altered lesions than in established carcinomas (46). In addition, MMP-2 was often expressed in non-tumorigenic ovarian cell lines, but reduced or absent in cancer cells. In the present study, alteration in both epithelial and stromal TIMP expressions was noted in benign compared to borderline or malignant tumors, either serous or mucinous. Strong stromal expressions of TIMP-1 and 2 were observed in benign tumors, while epithelial expression of TIMP-1, but not TIMP-2, was reduced in malignant ovarian tumors. Sakata et al also found high TIMP-1 expression and low TIMP-2 expression in benign and borderline tumors compared to malignant ovarian tumors (14). Määttä et al reported low TIMP-1 and 2 expressions in benign and borderline compared with malignant tumors (17). Because TIMPs are known inhibitors of MMP activities, their increased expression in tumor and stroma cells of malignant tumors observed in some studies seems paradoxical. However, TIMPs are also known to stimulate cell growth, like early erythroid progenitor cells or melanoma cells, or to activate proMMP-2 into MMP-2 by linkage between TIMP-2 and MT1-MMP $(8,47,48)$.

In conclusion, univariate analysis, hierarchical clustering, and MDS have demonstrated that serous and mucinous ovarian tumors have different MMP and TIMP expressions. This highlights the need to differentiate these tumors in evaluating their clinical, prognostic and therapeutic relevance to optimally select candidates for new targets.

\section{Acknowledgments}

The authors thank Miss Anita Rodenas for advice in setting up tissue microarray and for technical assistance in testing MMP antibodies for immunohistochemistry.

\section{References}

1. Hulboy DL, Rudolph LA and Matrisian LM: Matrix metalloproteinases as mediators of reproductive function. Mol Hum Reprod 3: 27-45, 1997.

2. Salamonsen LA and Nie G: Proteases at the endometrialtrophoblast interface: their role in implantation. Rev Endocr Metab Disord 3: 133-143, 2002.

3. Xue M, Le NT and Jackson CJ: Targeting matrix metalloproteases to improve cutaneous wound healing. Expert Opin Ther Targets 10: 143-155, 2006.

4. Chantrain CF, Henriet P, Jodele S, et al: Mechanisms of pericyte recruitment in tumour angiogenesis: a new role for metalloproteinases. Eur J Cancer 42: 310-318, 2006.

5. Curran S and Murray IG: Matrix metalloproteinases in tumour invasion and metastasis. J Pathol 189: 300-308, 1999.

6. Deryugina EI and Quigley JP: Matrix metalloproteinases and metastasis. Cancer Metastasis Rev 25: 9-34, 2006.

7. Liotta LA, Tryggvason K, Garbisa S, Hart I, Foltz CM and Shafie S: Metastatic potential correlates with enzymatic degradation of basement membrane collagen. Nature 284: 67-68, 1980 .

8. Strongin AY, Collier I, Bannikov G, Marmer BL, Grant GA and Goldberg GI: Mechanism of cell surface activation of $72-\mathrm{kDa}$ type IV collagenase. Isolation of the activated form of the membrane protease. J Biol Chem 270: 5331-5338, 1995.

9. Giannelli G, Falk-Marzillier J, Schiraldi O, Stetler-Stevenson WG and Quaranta V: Induction of cell migration by matrix metalloproteinase-2 cleavage of laminin-5. Science 277: 225-228, 1997.

10. Seiki M: Membrane-type 1 matrix metalloproteinase: a key enzyme for tumor invasion. Cancer Lett 194: 1-11, 2003.

11. Sounni NE and Noel A: Membrane type-matrix metalloproteinases and tumor progression. Biochimie 87: 329-342, 2005.

12. Pyke C, Ralfkiaer E, Tryggvason K and Dano K: Messenger RNA for two type IV collagenases is located in stromal cells in human colon cancer. Am J Pathol 142: 359-365, 1993.

13. Lokeshwar BL, Selzer MG, Block N and Gunja-Smith Z: Secretion matrix metalloproteinases and their inhibitors (tissue inhibitor of metalloproteinases) by human prostate in explant cultures: reduced tissue inhibitor of metalloproteinase secretion by malignant tissues. Cancer Res 53: 4493-4498, 1993. 
14. Sakata K, Shigemasa K, Nagai N and Ohama K: Expression of matrix metalloproteinases (MMP-2, MMP-9, MT1-MMP) and their inhibitors (TIMP-1, TIMP-2) in common epithelial tumors of the ovary. Int J Oncol 17: 673-681, 2000.

15. Wang FQ, So J, Reierstad S and Fishman DA: Matrilysin (MMP-7) promotes invasion of ovarian cancer cells by activation of progelatinase. Int J Cancer 114: 19-31, 2005.

16. Graesslin O, Cortez A, Fauvet R, Lorenzato M, Birembaut P and Darai E: Metalloproteinase-2, -7 and -9 and tissue inhibitor of metalloproteinase- 1 and -2 expressions in normal, hyperplastic and neoplastic endometrium: a clinical-pathological correlation study. Ann Oncol 17: 637-645, 2006.

17. Määttä M, Santala M, Soini Y, Talvensaari-Mattila A and Turpeenniemi-Hujanen T: Matrix metalloproteinases 2 and 9 and their tissue inhibitors in low malignant potential ovarian tumors. Tumour Biol 25: 188-192, 2004.

18. Kim TJ, Rho SB, Choi YL, et al: High expression of tissue inhibitor of metalloproteinase-2 in serous ovarian carcinomas and the role of this expression in ovarian tumorogenesis. Hum Pathol 37: 906-913, 2006.

19. Shigemasa K, Tanimoto H, Sakata K, et al: Induction of matrix metalloprotease-7 is common in mucinous ovarian tumors including early stage disease. Med Oncol 17: 52-58, 2000.

20. Benedet JL, Bender H, Jones H III, Ngan HYS and Pecorelli S: FIGO staging classifications and clinical practice guidelines in the management of gynecologic cancers. FIGO Committee on Gynecologic Oncology. Int J Gynecol Obstet 70: 209-262, 2000.

21. Kononen J, Bubendorf L, Kallioniemi A, et al: Tissue microarrays for high-throughput molecular profiling of tumor specimens. Nat Med 4: 844-847, 1998.

22. Skacel M, Skilton B, Pettay JD and Tubbs RR: Tissue microarrays: a powerful tool for high-throughput analysis of clinical specimen: a review of the method with validation data. Appl Immunohistochem Mol Morphol 10: 1-6, 2002

23. Budwit-Novotny DA, McCarty KS, Cox EB, et al: Immunohistochemical analyses of estrogen receptor in endometrial adenocarcinoma using a monoclonal antibody. Cancer Res 46: 5419-5425, 1986.

24. Eisen MB, Spellman PT, Brown PO and Botstein D: Cluster analysis and display of genome-wide expression patterns. Proc Natl Acad Sci USA 95: 14863-14868, 1998.

25. Liu CL, Prapong W, Natkunam Y, et al: Software tools for highthroughput analysis and archiving of immunohistochemistry staining data obtained with TMAs. Am J Pathol 161: 1557-1565, 2002.

26. Makretsov NA, Huntsman DG, Nielsen TO, et al: Hierarchical clustering analysis of tissue microarray immunostaining data identifies prognostically significant groups of breast carcinoma. Clin Cancer Res 10: 6143-6151,2004.

27. Sorlie T, Perou CM, Tibshirani R, et al: Gene expression patterns of breast carcinomas distinguish tumor subclasses with clinical implications. Proc Natl Acad Sci USA 98: 10869-10874, 2001.

28. Rouzier R, Perou CM, Symmans WF, et al: Breast cancer molecular subtypes respond differently to preoperative chemotherapy. Clin Cancer Res 11: 5678-5685, 2005.

29. Feeley KM and Wells M: Precursor lesions of ovarian epithelial malignancy. Histopathology 38: 87-95, 2001

30. Tresserra F, Grases PJ, Labatisda R and Ubeda A: Histological features of the contralateral ovary in patients with unilateral ovarian cancer: a case control study. Gynecol Oncol 71: 437-441, 1998.

31. Werness BA, Afify AM, Bielat KL, Eltabbakh GH, Piver MS and Paterson JM: Altered surface and cyst epithelium of ovaries removed prophylactically from women with a family history of ovarian cancer. Hum Pathol 30: 151-157, 1999.
32. Fauvet R, Dufournet C, Poncelet C, Uzan C, Hugol D and Darai E Expression of pro-apoptotic (p53, p21, bax, bak and fas) and anti-apoptotic (bcl-2 and bcl-x) proteins in serous versus mucinous borderline ovarian tumours. J Surg Oncol 92: 337-343, 2005.

33. Afzal S, Lalani EN, Poulsom R, et al: MT1-MMP and MMP-2 mRNA expression in human ovarian tumors: possible implications for the role of desmoplastic fibroblasts. Hum Pathol 29: $155-165,1998$

34. Wu M, Xu G, Xi L, et al: Down-regulation of MT1-MMP expression suppresses tumor cell invasion in metastatic human SW626 ovarian cancer cells. Oncol Rep 15: 501-505, 2006.

35. Sodek KL, Ringuette MJ and Brown TJ: MT1-MMP is the critical determinant of matrix degradation and invasion by ovarian cancer cells. Br J Cancer 97: 358-367, 2007.

36. Sillanpää SM, Antilla MA, Voutilainen KA, et al: Prognostic significance of matrix metalloproteinase-7 in epithelial ovarian cancer and its relation to ß-catenin. Int J Cancer 119: 1792-1799, 2006.

37. Behrens P, Rothe M, Florin A, Wellman A and Wernert N Invasive properties of serous human epithelial ovarian tumors are related to Ets-1, MMP-1 and MMP-9 expression. Int J Mol Med 8: 149-154, 2001.

38. Ozalp S, Tanir HM, Yalcin OT, Kabukcuoglu S, Oner U and Uray M: Prognostic value of matrix metalloproteinase- 9 (gelatinase B) expression in epithelial ovarian tumors. Eur J Gynaecol Oncol 24: 417-420, 2003.

39. Zhou HY, Pon YL and Wong AS: Synergistic effects of epidermal growth factor and hepatocyte growth factor on human ovarian cancer cell invasion and migration: role of extracellular signalregulated kinase $1 / 2$ and p38 mitogen-activated protein kinase. Endocrinology 148: 5195-5208, 2007.

40. Cowden Dahl KD, Zeineldin R and Hudson LG: PEA3 is necessary for optimal epidermal growth factor receptorstimulated matrix metalloproteinase expression and invasion of ovarian tumor cells. Mol Cancer Res 5: 413-421, 2007.

41. Symowicz J, Adley BP, Gleason KJ, et al: Engagement of collagen-binding integrins promotes matrix metalloproteinase9-dependent E-cadherin ectodomain shedding in ovarian carcinoma cells. Cancer Res 67: 2030-2039, 2007.

42. Darai E, Scoazec JY, Walker-Combrouze F, et al: Expression of cadherins in benign, borderline, and malignant ovarian epithelial tumors: a clinicopathological study of 60 cases. Hum Pathol 28: 922-928, 1997.

43. Westerlund A, Apaja-Sarkkinen M, Höyhtyä M, Puistola U and Turpeenniemi-Hujanen T: Gelatinase A-immunoreactive protein in ovarian lesions: prognostic value in epithelial ovarian cancer. Gynecol Oncol 75: 91-98, 1999.

44. Wu X, Li H, Kang L, Wang W and Shan B: Activated matrix metalloproteinase-2: a potential marker of prognosis for epithelial ovarian cancer. Gynecol Oncol 84: 126-134, 2002.

45. Schmalfeldt B, Prechtel D, Härting K, et al: Increased expression of matrix metalloproteinases (MMP)-2, MMP-9, and the urokinase-type plasminogen activator is associated with progression from benign to advanced ovarian carcinoma. Clin Cancer Res 7: 2396-2404, 2001

46. Qi Cai K, Yang WL, Capo-Chichi CD, et al: Prominent expression of metalloproteinases in early stages of ovarian tumorigenesis. Mol Carcinog 46: 130-143, 2007.

47. Chesler L, Golde DW, Bersch N and Johnson MD: Metalloproteinase inhibition and erythroid potentiation are independent activities of tissue inhibitor of metalloproteinases-1. Blood 86: 4506-4515, 1995

48. Hofmann UB, Houben R, Bröcker EB and Becker JC: Role of matrix metalloproteinases in melanoma cell invasion. Biochimie 87: 307-314, 2005. 